\title{
Sangrado digestivo alto secundario a tumores del estroma gastrointestinal (GIST) asociado a Linfoma MALT: caso clínico
}

\author{
Upper gastrointestinal bleeding secondary to gastrointestinal stromal tumors \\ (GIST) associated with MALT lymphoma: clinical case
}

Sangramento gastrointestinal superior secundário a tumores estromal gastrointestinal (GIST) associado a Linfoma MALT: caso clínico

\author{
Marcelo Castillo Paucar (iD ${ }^{a}$, Gabriela Fernanda Quingalombo Cargua ${ }^{\text {iD }}{ }^{\text {b }}$, Tania Paulina \\ Garcés García (iD ${ }^{\text {c }}$
}

\begin{abstract}
a Hospital de Especialidades Eugenio Espejo, Quito-Ecuador; b Pontificia Universidad Católica del Ecuador, Hospital de Especialidades Eugenio Espejo, Quito-Ecuador: c Universidad Central del Ecuador, Hospital de Especialidades Eugenio Espejo, Quito-Ecuador.
\end{abstract}

Correspondencia a:

Marcelo Castillo Paucar, drchelocastillo@gmail.com

Recibido: 15 de octubre, 2021

Aceptado: 19 de noviembre, 2020 Publicado: 4 de enero, 2021

\section{CASO CLÍNICO}

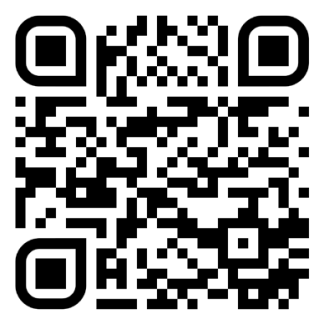

Escanea en tu dispositivo móvil o revisa este artículo en: https:// revistaclinicaguayaquil.org

\section{RESUMEN}

Los tumores del estroma gastrointestinal (GIST) son neoplasias raras que se localizan predominantemente en el estómago; sus síntomas son sangrado gastrointestinal, anemia y dolor abdominal. Se diagnostica en presencia de positividad del proto oncogén KIT o CD34, desmina, S-100, DOG1, entre otros. El tratamiento es la resección en cuña o imatinib según el estadio. El Linfoma MALT es un Linfoma no Hodgkin extra nodal de estirpe B que se localiza en el estómago frecuentemente. Su sintomatología suele ser inespecífica: náuseas, vómitos, anorexia, pérdida de peso y sangrado. Su diagnóstico requiere la presencia de células plasmáticas, folículos reactivos y células tipo centrocitos que invaden la mucosa y forman lesiones linfoepitaliales; son positivos para CD20, CD10, CD5 y ciclina D1. Las neoplasias sincrónicas gástricas son pocos frecuentes, el diagnóstico generalmente ocurre de forma incidental durante el estudio histopatológico de una pieza quirúrgica. Estos tumores representan un reto clínico para su tratamiento, ya que cada uno debe ser estadificado por separado. Se presenta el caso clínico de un paciente de 31 años, quien consulta por hematemesis y melenas, con antecedentes de lesión subcardial subepitelial en endoscopía previa, con marcadores tumorales negativos y estudio histopatológico que demostró un tumor del estroma gastrointestinal tipo de células fusiformes asociado a Linfoma no Hodgkin; quien recibió tratamiento erradicador para $\mathrm{H}$. pylori e Imatinib con adecuada respuesta.

Palabras clave: Linfoma; tumores del estroma gastrointestinal; linfoma del tejido linfoide asociado a mucosa

\begin{abstract}
Gastrointestinal stromal tumors (GIST) are rare neoplasms that are predominantly located in the stomach; its symptoms include gastrointestinal bleeding, anemia, and abdominal pain. It is diagnosed in the presence of positivity of KIT or CD34 protooncogene, desmin, S-100, DOG1, among others. Treatment is wedge resection or imatinib depending on its stage. MALT Lymphoma is a B-line extranodal non-Hodgkin Lymphoma that is frequently located in the stomach; its symptoms are usually nonspecific: nausea, vomiting, anorexia, weight loss and bleeding. Its diagnosis requires the presence of plasma cells, reactive follicles, and centrocyte-like cells that invade the mucosa and form lymphoepitalial lesions. They are positive for CD20, CD10, CD5, and cyclin D1. Gastric synchronous neoplasms are rare, the diagnosis generally occurs incidentally during the histopathological study of a surgical piece. These tumors represent a clinical challenge for their treatment, since each one must be staged separately.
\end{abstract}


Key words: Lymphoma; gastrointestinal stromal tumors; mucosa-associated lymphoid tissue

\section{RESUMO}

Os tumores estromais gastrointestinais (GIST) são neoplasias raras que estão predominantemente localizadas no estômago; seus sintomas são sangramento gastrointestinal, anemia e dor abdominal. É diagnosticado na presença de positividade do proto-oncogene KIT ou CD34, desmina, S-100, DOG1, entre outros. O tratamento é a ressecção em cunha ou imatinibe, dependendo do estágio. O Linfoma MALT é um Linfoma não-Hodgkin extranodal de linha $B$, frequentemente localizado no estômago. Seus sintomas geralmente são inespecíficos: náuseas, vômitos, anorexia, perda de peso e sangramento. Seu diagnóstico requer a presença de células plasmáticas, folículos reativos e células semelhantes a centrócitos que invadem a mucosa e formam lesões linfoepitaliais; eles são positivos para CD20, CD10, CD5 e ciclina D1. As neoplasias síncronas gástricas são raras, o diagnóstico geralmente ocorre incidentalmente durante o estudo histopatológico de uma peça cirúrgica. Esses tumores representam um desafio clínico para o seu tratamento, uma vez que cada um deve ser estadiado separadamente. Apresentamos o caso clínico de uma paciente de 31 anos, que se consultou por hematêmese e melena, com história de lesão subepitelial subcardial em endoscopia prévia, com marcadores tumorais negativos e estudo histopatológico que demonstrou tumor estromal gastrointestinal tipo fusocelular associado a linfoma, não Hodgkin; que receberam tratamento de erradicação para H. pylori e Imatinibe com resposta adequada.

Palavras-chave: Linfoma; Tumores Do Estroma Gastrointestinal; tecido linfóide associado à mucosa

\section{INTRODUCCIÓN}

Los tumores del estroma gastrointestinal (GIST) son tumores poco comunes que comprometen predominantemente al estómago (50-62\%), intestino delgado (20-30\%), colon (11\%) y el recto (7\%). La coexistencia con otras neoplasias oscila entre el 3 al $33 \%$, siendo los adenocarcinomas los más comunes y en menor grado el linfoma, leucemia, carcinoma de mama, próstata, páncreas, pulmón o adenomas adrenocorticales (1-3).

Los tumores que se desarrollan desde diferentes partes del estómago con la misma o distinta histología concurrente son llamados tumores sincrónicos. El GIST y linfoma concomitante en el estómago es un fenómeno raro, no está claro si comparten la misma etiología o no (4). El estómago es el sitio más frecuente de linfoma digestivo; la mayoría de estos linfomas se clasifican como linfoma de los linfocitos B de la zona marginal del tejido linfático asociado a la mucosa (MALT) o como linfoma difuso de linfocitos B grandes (5). Los GIST comúnmente se presentan como lesiones elevadas que pueden ulcerar la mucosa que los rodea y ser causa de hemorragia gastrointestinal aguda. Entender los fenómenos fisiopatológicos de esta rara entidad nos puede ayudar a mejorar su diagnóstico y prevenir o tratar sus complicaciones, principalmente el sangrado digestivo alto $(6,7)$.

\section{DESCRIPCIÓN DEL CASO CLÍNICO}

Paciente masculino de 31 años, con antecedente de psoriasis en tratamiento tópico, como hábitos perniciosos reportó consumo de cocaína hace 10 años y actual de tabaco y marihuana. Acudió a emergencias por presentar hematemesis y melenas por tres ocasiones, sin presentar otra sintomatología. Se realizó una endoscopia digestiva alta (VEDA) en la que se evidenció un tumor submucoso de $5 \mathrm{~cm}$ subcardial con úlcera tunelizada (figura 1). En ausencia de signos de sangrado digestivo es dado de alta con inhibidores de la bomba de protones. A las 48 horas presentó nuevo episodio de melena y hematemesis, presentando en los laboratorios una hemoglobina de $11 \mathrm{~g} / \mathrm{dl}$, hematocrito $33.1 \%$, plaquetas $531.000 \mathrm{k} / \mathrm{ul}$, tiempo de protombina 14.9 segundos, tiempo parcial de tromboplastina 14.90 segundos, INR 1.30. La VEDA reportó una lesión subcardial subepitelial de $6 \mathrm{~cm}$ de diámetro, con úlcera central sin signos de sangrado activo, localizada en región subcardial, a $3 \mathrm{~cm}$ del hiato a nivel de curvatura. Por sospecha de etiología neoplásica se realizó una tomografía de abdomen la cual mostró engrosamiento de la curvatura menor e imágenes hepáticas sugestivas de metástasis. Los marcadores tumorales fueron negativos. La ecoendoscopia evidenció una lesión hipo ecogénica bien delimitada que mide $7 \times 12$ $\mathrm{mm}$, localizada en la muscular propia (figura 2). El paciente no presentó nuevos episodios de sangrado.

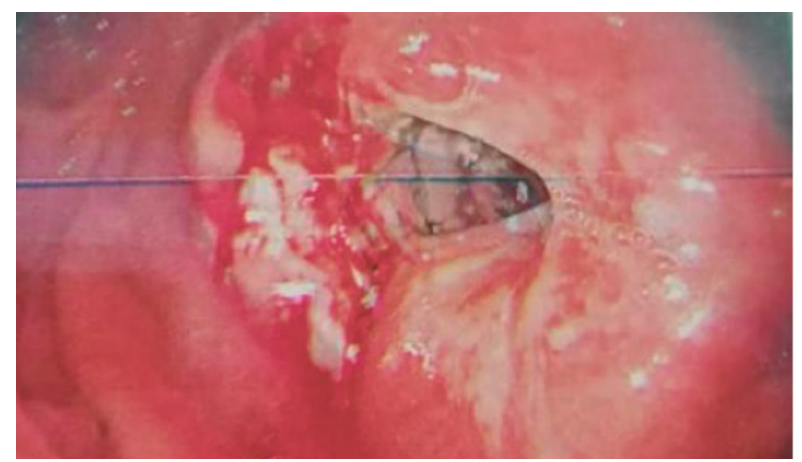

Figura 1. Video endoscopia digestiva alta en la que se evidenció un tumor submucoso de $5 \mathrm{~cm}$ subcardial con úlcera tunelizada. 


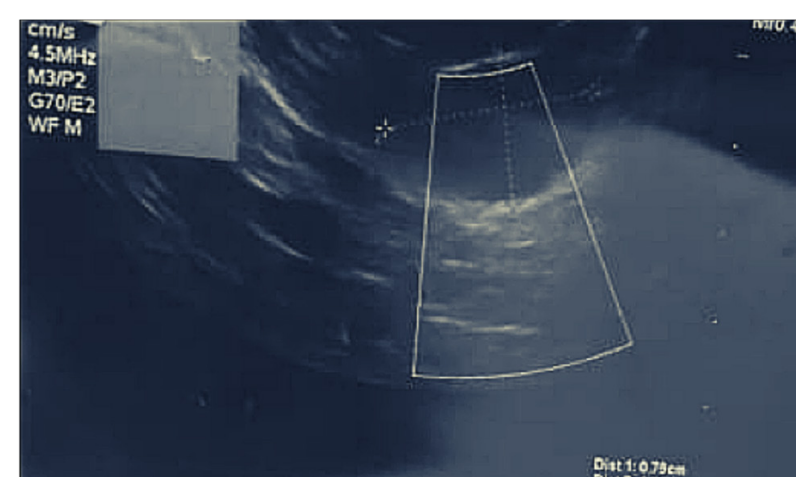

Figura 2. Endoscopía en la que se observa lesión hipoecogénica, bien delimitada de 7 × $12 \mathrm{~mm}$, localizada en la muscular propia.

El resultado histopatológico informó cortes con tinción de hematoxilina eosina con neoplasia mesenquimal constituido por células fusiformes de núcleos alargados, proliferación de linfocitos atípicos de tamaño intermedio a grande y el perfil inmunohistoquímico CD117 y DOG 1 positivos en células neoplásicas, actina y S100 negativo en células neoplásicas; hallazgos que son compatibles con GIST asociado a MALT con Helicobacter pylori negativo.

El paciente recibe tratamiento con Imatinib 400 mg/día (inhibidor de la tirosin kinasa), además recibió tratamiento de erradicación para $H$. pylori con terapia de primera línea. Así también, debido a las lesiones hepáticas sugestivas de metástasis, se sugirió realizar biopsia hepática, procedimiento rechazado por el paciente.

En los posteriores controles tomográficos y endoscópicos, al momento de la presente publicación, se ha evidenciado disminución del tamaño de las lesiones; el paciente se encuentra clínicamente estable, con un año de tratamiento con Imatinib.

\section{DISCUSIÓN}

Se denomina neoplasias malignas primarias múltiples (NMPM) a dos o más tumores malignos distintos, que se generan en un mismo paciente. Las neoplasias sincrónicas son pocos frecuentes; recientemente se han publicado casos de GIST de manera simultánea con otras neoplasias malignas (8). Los GIST reportados de manera sincrónica con otras neoplasias del tracto gastrointestinal incluyen: adenocarcinomas gástricos, linfomas, cánceres de esófago, colon, páncreas, hepatocarcinoma y tumores carcinoides. La asociación sincrónica más frecuente es con adenocarcinomas gástricos, correspondiendo al $47 \%$ de los casos de sincronismo (9). El diagnóstico de tumores gástricos simultáneos generalmente ocurre de forma incidental durante el estudio histopatológico de una pieza quirúrgica. Representan un reto clínico para su tratamiento, ya que cada uno de estos tumores debe ser estadificado por separado, debido a que tienen esquemas de tratamiento diferentes.

La coexistencia del adenocarcinoma gástrico y Linfoma MALT está bien documentada. Se sugiere que la secuencia de cambios que ocurren desde gastritis crónica hasta displasia, eventualmente puede desencadenar un adenocarcinoma gástrico debido a los efectos de la acumulación de compuestos $\mathrm{N}$-nitroso secundarios a infección por Helicobacter pylori. Sin embargo, en el caso de sincronismo entre un GIST y Linfoma MALT, la relación hasta el momento no es clara, por lo que se requieren más estudios para conocer si existe un factor causal entre los GIST y el Linfoma MALT (10).

Los principales patrones histológicos de los tumores del estroma son: tipo de células en forma de huso (60-70\%), tipo de células epiteliales (30-40\%) (11) y tipo mixto (10\%) (12).

Los síntomas más comunes de los GIST son el sangrado gastrointestinal, anemia y dolor abdominal, con menor frecuencia distensión abdominal y malestar debido al efecto de masa producido por el tumor, puede incluso presentarse como rotura tumoral, perforación u obstrucción $(12,13)$. No obstante, entre el 15\% y $30 \%$ de los pacientes pueden ser asintomáticos (12), y el $5 \%$ se encuentran incidentalmente durante la autopsia (13).

LoS GIST se diagnostican en presencia de positividad KIT o CD34. Si el tumor es negativo para KIT, CD34, desmina y S-100, las pruebas adicionales consisten en la tinción DOG1 o una mutación del gen KIT o PDGFRA para su diagnóstico (12). Otras moléculas como vimentina, actina del músculo liso (SMA), neurofilamento (NF), enolasa específica de neuronas (NSE) ayudan al diagnóstico (11). La endoscopia proporciona datos útiles sobre la localización y naturaleza de la lesión y revela una lesión subepitelial cubierta con mucosa normal, otras veces tiene bordes irregulares y ulceración considerando estas características clínicamente malignas $(12,14)$. La tomografía computarizada se realiza para la estadificación, mientras que la ecoendoscopia define la profundidad de la lesión, observándose como una masa sólida hipoecoica que compromete hasta la muscular propia correspondiente a la cuarta eco capa $(12,14)$. 
El tratamiento de los tumores localizados es la resección en cuña del estómago, mientras que para la enfermedad no resecable y/o metastásica, el tratamiento con Imatinib es la primera opción y su periodo de respuesta es de 12 a 36 meses (14).

El diagnóstico de Linfoma MALT gástrico se basa en el examen histopatológico de acuerdo a la clasificación de la Organización Mundial de la Salud (OMS), y se caracteriza por la presencia de células plasmáticas, folículos reactivos y células tipo centrocitos que invaden la mucosa y forman las lesiones linfoepitaliales características (6). Su diferenciación de otros linfomas indolentes no es siempre sencilla y debería incluir un panel inmuno histoquímica mínimo con CD20, CD10, CD5 y ciclina D1. Si la infección por H. pylori no es demostrada por histoquímica, se debe descartar con test de aliento de urea, serología y/o antígeno en heces (1).

Los síntomas del Linfoma MALT no son específicos e incluyen náuseas, vómitos, anorexia y pérdida de peso, el sangrado ocurre en $20 \%$ de los casos. De acuerdo al sistema de estadiaje de Lugano, el estadio IE1 se refiere a la infiltración de la mucosa y submucosa, mientras el IE2 se extiende más allá de la submucosa (3). Para los estadios IE1, IE2 o IIE con H. Pylori positivo, se debe dar tratamiento para la bacteria y evaluar su erradicación con endoscopia. Para los estadios IE y IIE con $\mathrm{H}$. Pylori negativo, el tratamiento consiste en radioterapia o Rituximab. En estadios avanzados (IIIE y IV) se debe determinar si tiene o no indicación para tratamiento, de lo contrario el manejo es conservador; las indicaciones para tratar son la presencia de síntomas, hemorragia gastrointestinal, enfermedad de Bulky, preferencia del paciente, progresión del estadiaje, y daño de órgano. Las opciones de tratamiento incluyen quimio inmunoterapia, radioterapia y enrolamiento en ensayos clínicos. La cirugía es reservada para casos específicos donde está en riesgo la vida por hemorragia (15).

En relación a nuestro paciente se estableció el diagnóstico de tumor sincrónico gastrointestinal GIST + Linfoma no Hodgkin asociado a H. pylori + lesiones metastásicas hepáticas de GIST. Se decidió iniciar tratamiento con Imatinib y terapia de primera línea para $\mathrm{H}$. pylori. Por el momento no se ha considerado tratamiento quirúrgico para el GIST por no presentar signos de urgencia como sangrado o inadecuada respuesta al tratamiento.

\section{REFERENCIAS BIBLIOGRÁFICAS}

1. Herrera-Goepfert R, Lizano M, Akiba S, Carrillo-1. Casali PG, Abecassis N, Aro HT, Bauer S, Biagini R, Bielack S, et al. Gastrointestinal stromal tumours: ESMO-EURACAN Clinical Practice Guidelines for diagnosis, treatment and follow-up. Ann Oncol Off J Eur Soc Med Oncol. 2018;29(Suppl 4):iv6878. doi: 10.1093/annonc/mdy095.

2. Kaur R, Bhalla S, Nundy S, Jain S. Synchronous gastric gastrointestinal stromal tumor (GIST) and other primary neoplasms of gastrointestinal tract: report of two cases. Annals of gastroenterology. 2013;26(4):356-9.

3. Samaras VD, Foukas PG, Triantafyllou K, Leontara V, Tsapralis D, Tsompanidi EM, et al. Synchronous well differentiated neuroendocrine tumour and gastrointestinal stromal tumour of the stomach: a case report. BMC Gastroenterol. 2011;11(1):27. doi: 10.1186/1471-230X-11-27.

4. Pamukçuoglu M, Budakoğlu B, Han O, Tad M, Oksüzoğlu B, Abali $\mathrm{H}$, et al. An extraordinary case in whom gastrointestinal stromal tumor and lowgrade malignant lymphoma are seen together in the stomach. Med Oncol. 2007;24(3):351-3. doi: 10.1007/s12032-007-0001-4.

5. Feldman M. Tumores del estroma gastrointestinal (TEG). En: Feldman M, Friedman L, Brandt L, editores. Enfermedades digestivas y hepáticas. 10ma ed. Elsevier; 2017. p. 487-500.

6. Wai K, Enders $K$, Joseph J. Tumors of the stomach. En: Tadataka Y, David H, Antony $N$, editores. Textbook of Gastroenterology. 5ta ed. UK: Blackwell Publishing; 2019. p. 1026-54.

7. Zucca E, Copie-Bergman C, Ricardi U, Thieblemont C, Raderer M, Ladetto M. Gastric marginal zone lymphoma of MALT type: ESMO Clinical Practice Guidelines for diagnosis, treatment and followup. Ann Oncol. 2013;24(Suppl 6):vi144-8. doi: 10.1093/annonc/mdt343.

8. Ladrón De Guevara D, Quera R, Rozas S, Schacher S, Reyes JM, Pardo C, et al. Cáncer sincrónico y metacrónico detectado con PET/CT en población oncológica. Rev Med Chil. 2017;145(11):1421-8. doi: 10.4067/s0034-98872017001101421.

9. Guerra Mesa JL, Lamadrid García J, Ruíz Calderón I. Adenocarcinoma gástrico y tumor estromal del estómago sincrónico incidental. Revista Cubana de Cirugía. 2017;56(4):1-11.

10. Martínez-Berlanga P, González-Acosta J, KuriOsorio J, Vega de Jesús M, Medina-LeónJ. Presencia simultánea de adenocarcinoma gástrico, linfoma de tejido linfoide asociado a la mucosa gástrica y tumor de estroma gastrointestinal: reporte de un caso. Rev Sanid Milit. 2011;65(1):32-5.

11. Graadt van Roggen JF, van Velthuysen ML, Hogendoorn PC. The histopathological differential diagnosis of gastrointestinal stromal tumours. J Clin Pathol. 2001;54(2):96-102. doi: 10.1136/ jcp.54.2.96.

12. Akahoshi K, Oya M, Koga T, Shiratsuchi Y. Current 
clinical management of gastrointestinal stromal tumor. World J Gastroenterol. 2018;24(26):280617. doi: 10.3748/wjg.v24.i26.2806.

13. von Mehren M, Joensuu H. Gastrointestinal Stromal Tumors. J Clin Oncol. 2018;36(2):136-43. doi: 10.1200/JCO.2017.74.9705.

14. Akahoshi K, Oya M. Gastrointestinal stromal tumor of the stomach: How to manage? World
J Gastrointest Endosc. 2010;2(8):271-7. doi: 10.4253/wjge.v2.i8.271.

15. National Comprehensive Cancer Network. NCCN Clinical Practice Guidelines in Non-Hodgkin's Lymphomas [Internet]. Fort Washington, PA; 2015. Disponible en: https://www2.tri-kobe.org/ nccn/guideline/hematologic/nhl/english/foll.pdf

\section{ACERCA DE LOS AUTORES}

1. Marcelo Castillo Paucar. Especialista en Medicina Interna por la PUCE. Egresado de la subespecialidad en Gastroenterología y Endoscopia por la UCE. Docente de la cátedra de Medicina Interna de la facultad de Odontologia de la UTE. Exdocente de primer año de la Escuela de Medicina de la PUCE. Hospital de Especialidades Eugenio Espejo, Quito-Ecuador.

ORCID: 0000-0002-9033-4148

2. Gabriela Fernanda Quingalombo Cargua. Médica por la Universidad Central del Ecuador, Especialidad en Medicina Familiar y Comunitaria por la Pontificia Universidad Católica del Ecuador con beca del Hospital Vozandes, actualmente posgradista del quinto semestres de Gastroenterología y Endoscopía de la Pontificia Universidad Católica del Ecuador, Ecuador.

ORCID: 0000-0001-6132-1527

3. Tania Paulina Garcés García. Médico especialista en anatomía patológica. Maestría en gerencia en salud para el desarrollo local. Médico tratante del hospital de especialidades Eugenio Espejo. Médico tratante en laboratorio de patología "GASTROPATH". Docente del post-grado de patología de la Universidad Central del Ecuador. Extutora del postgrado de medicina legal de la Universidad Central del Ecuador. Exdocente de la cátedra de Histología de Pregrado de la Universidad San Francisco de Quito. Hospital de Especialidades Eugenio Espejo, Ecuador.

ORCID: 0000-0003-1869-4277 